\title{
Solving Electric Market Quadratic Problems by Branch and Fix Coordination Methods ${ }^{\star}$
}

\author{
F.-Javier Heredia ${ }^{1}$, Cristina Corchero ${ }^{1}$, and Eugenio Mijangos ${ }^{2}$ \\ 1 Department of Statistics and Operations Research \\ Universitat Politècnica de Catalunya UPC \\ \{f.javier.heredia, cristina.corchero\}@upc.edu \\ 2 Department of Applied Mathematics, \\ Statistics and Operations Research \\ University of the Basque Country UPV/EHU \\ eugenio.mijangos@ehu.es
}

\begin{abstract}
The electric market regulation in Spain (MIBEL) establishes the rules for bilateral and futures contracts in the day-ahead optimal bid problem. Our model allows a price-taker generation company to decide the unit commitment of the thermal units, the economic dispatch of the bilateral and futures contracts between the thermal units and the optimal sale bids for the thermal units observing the MIBEL regulation. The uncertainty of the spot prices is represented through scenario sets. We solve this model on the framework of the Branch and Fix Coordination metodology as a quadratic two-stage stochastic problem. In order to gain computational efficiency, we use scenario clusters and propose to use perspective cuts. Numerical results are reported.
\end{abstract}

Keywords: Liberalized Electricity Market, Optimal Bid, Stochastic Programming, Quadratic Branch-and-Fix Coordination.

\section{Introduction}

This work is applied to the Iberian Electricity Market (MIBEL) comprising the Spanish and Portuguese electricity systems. The MIBEL market includes in the short-term: the day-ahead market (DAM) and a set of balancing, reserve and adjustment markets (intraday markets); these markets are complemented with the medium- and long-term mechanisms: a derivatives market and different kinds of bilateral contracts. This structure is similar to other European electricity markets and explains why generation companies can no longer optimize their short-term generation planning decisions, i.e. their bidding strategies, without considering the relationship between the short-term bid and the medium-term physical products. The MIBEL's directives dictate specific rules describing how these medium-term mechanisms should be included into the DAM bid. This work deals with the most relevant medium-term mechanisms in the MIBEL:

\footnotetext{
* This work was partially supported by the Ministry of Science and Technology of Spain through MICINN Project DPI2008-02153.
} 
the national bilateral contracts (BC) and the future physical contracts (FC). Stochastic programming techniques are applied to maximize the expected value of the utility's profit coming from the day-ahead market, where the significative random variable is the auction clearing price of the day-ahead electricity market. This random variable is modeled through a set of scenarios of the forecasted prices. The set of scenarios is used to feed a two-stage stochastic optimization model that finds the optimal day-ahead bid of a price-taker GenCo (an electrical utility without influence over the market prices) operating in the MIBEL and holding bilateral and physical futures contracts.

The extensive form of the deterministic equivalent of this stochastic programming problem will be a mixed integer quadratic programming problem (MIQP), which is difficult to solve efficiently, particularly for large-scale instances. Several algorithmic approaches can be adopted to overcome this difficulty. In 2$]$ the quadratic objective function of this problem is approximated by a polyhedral outer approximation by means of perspective cuts so that we can exploit the efficiency of general-purpose solvers for mixed integer linear problems (MILP). An alternative to the perspective cuts methodology is the Second-Order Cone Program reformulation (SOCP, [9]), but for quadratic problems the perspective cuts reformulation was reported to be more efficient [6]. Finally, the Branch-andFix Coordination (BFC) method has been used successfully to solve two-stage stochastic mixed integer linear problems 3 . to solve the day-ahead optimal bid problem. In this work we propose an combination between BFC and PC to efficiently solve the optimal day-ahead bid problem.

\section{Day-ahead Electricity Market Bid with Futures and Bilateral Contracts Model (DAMB-FBC)}

In this section the model (DAMB-FBC) is formulated as a two-stage stochastic programming problem that allows a price-taker generation company to optimally decide the unit commitment of its thermal units, the economic dispatch of the bilateral and futures contracts between the thermal units, and the optimal generation bid of the committed units to the MIBEL's day-ahead market. The objective function of the model represents the expected profits of the GenCo's participation in the day-Ahead market. The constraints assure that the MIBEL's rules and the operational restrictions of the units are respected. The main decision variables are the ones that model the start-up and shut-down of the units, the quantity that will be bid at instrumental price and the scheduled energy committed to the bilateral and the futures contracts settlement.

\subsection{Parameters}

The (DAMB-FBC) model considers a price-taker GenCo owning a set of thermal generation units $\mathcal{I}$ that bid to the $t \in \mathcal{T}=\{1,2, . ., 24\}$ hourly auctions of the DAM. The parameters for the $i^{\text {th }}$ thermal unit are: 
$-c_{i}^{b}, c_{i}^{l}$ and $c_{i}^{q}$, generation costs with constant, linear and quadratic coefficients (€, €/MWh and $€ / \mathrm{MWh}^{2}$ respectively).

- $\bar{P}_{i}$ and $\underline{P}_{i}$, upper and lower bounds on the hourly energy generation (MWh).

$-c_{i}^{o n}$ and $c_{i}^{\text {off }}$, start-up and shut-down costs $(€)$.

- $t_{i}^{o n}$ and $t_{i}^{\text {off }}$, minimum operation and minimum idle time (h).

A base load physical futures contract $j \in \mathcal{F}$ is defined by:

$-\mathcal{U}_{j}$, the set of generation units allowed to cover the $\mathrm{FC} j$.

- $L_{j}^{F}$, the amount of energy (MWh) to be procured each interval of the delivery period by the set $\mathcal{U}_{j}$ of generation units to cover contract $j$.

$-\lambda_{j}^{F}$, the price of contract $j(€ / \mathrm{MWh})$.

A base load bilateral contract $k \in \mathcal{B}$ is defined by:

- $L_{k}^{B}$, the amount of energy ( $\mathrm{MWh}$ ) to be procured at each interval of the delivery period by the set of available generation units to cover the BCs.

$-\lambda_{k}^{B}$, the price of the contract $k(€ / \mathrm{MWh})$.

The random variable $\lambda_{t}^{D}$, the clearing price of the $t^{\text {th }}$ hourly auction of the DAM, is represented in the two-stage stochastic model by a set of scenarios $s \in \mathcal{S}$, each one with its associated clearing price for each DAM auction $t \in \mathcal{T}$ :

$-\lambda_{t}^{D, s}$ clearing price for auction $t$ at scenario $s(€ / \mathrm{MWh})$.

- $P^{s}$ probability of scenario $s$.

\section{$2.2 \quad$ Variables}

Those decision variables that doesn't depend on the scenarios are called first stage (or here-and-now) variables and in our formulation are, for each $t \in \mathcal{T}$ and $i \in \mathcal{I}$ :

- $u_{t i}$, the unit commitment (binary)

$-c_{t i}^{u}, c_{t i}^{d}$, the start-up/shut-down costs variables.

- $q_{t i}$, the instrumental price offer bid.

- $f_{t i j}$, the scheduled energy for FC $j \in \mathcal{F}$.

$-b_{t i}$, the scheduled energy for the pool of BCs .

Decision variables that can adopt different values depending on the scenario are called second stage variables and in our formulation are, for each $t \in \mathcal{T}, i \in \mathcal{I}$ and scenario $s \in \mathcal{S}$ :

$-g_{t i}^{s}$, the total generation.

$-p_{t i}^{s}$, the matched energy in the day-ahead market. 


\subsection{Contraints}

Bilateral and Futures Contracts Constraints. The coverage of both the physical futures contracts and the bilateral contracts must be guaranteed. The constraints for each futures contract are:

$$
\begin{array}{ll}
\sum_{i \in \mathcal{U}_{j}} f_{t i j}=L_{j}^{F} & t \in \mathcal{T}, j \in \mathcal{F} \\
f_{t i j} \geq 0 & t \in \mathcal{T}, j \in \mathcal{F}, i \in \mathcal{I}
\end{array}
$$

and the bilateral contract constraints are:

$$
\begin{array}{ll}
\sum_{i \in \mathcal{I}} b_{t i}=\sum_{k \in \mathcal{B}} L_{k}^{B} & t \in \mathcal{T} \\
0 \leq b_{t i} \leq \bar{P}_{i} u_{t i} & i \in \mathcal{I}, t \in \mathcal{T}
\end{array}
$$

where $L_{k}^{B}$ is the energy that has to be settled for contract $k \in \mathcal{B}$

Day-ahead Market and Total Generation Constraints. As we have introduced, we will use the value of the matched energy in our formulation. The matched energy is the accepted energy in the clearing process, that is, the energy generated that will be rewarded at the clearing price. This matched energy is uniquely determined by the sale bid and the clearing price and it will play a central role in the presented model [2].

The MIBEL's rules affecting the day-ahead market establishes the relation between the variables representing the matched energy $p_{t i}^{s}$, the energy of the bilateral contracts $b_{t i}$, the energy of the futures contracts $f_{t i j}$, the instrumental price offer bid $q_{t i}$, and the commitment binary variables $u_{t i}$. The energies $L_{j}^{F}$ and $L_{k}^{B}$ must be integrated in the MIBEL's DAM bid observing the two following rules:

1. If generator $i$ contributes with $f_{t i j}$ MWh at period $t$ to the coverage of the FC $j$, then the energy $f_{t i j}$ must be offered to the pool for free (instrumental price bid).

2. If generator $i$ contributes with $b_{t i} \mathrm{MWh}$ at period $t$ to the coverage of any of the BCs, then the remaining production capacity $\bar{P}_{i}-b_{t i}$ must be bid to the DAM.

These rules can be included in the model by means of the following set of constraints:

$$
\begin{array}{ll}
p_{t i}^{s} \geq q_{t i} & i \in \mathcal{I}, t \in \mathcal{T}, s \in \mathcal{S} \\
p_{t i}^{s} \leq \bar{P}_{i} u_{t i}-b_{t i} & i \in \mathcal{I}, t \in \mathcal{T}, s \in \mathcal{S} \\
q_{t i} \geq \underline{P}_{i} u_{t i}-b_{t i} & i \in \mathcal{I}, t \in \mathcal{T}, s \in \mathcal{S} \\
q_{t i} \geq \sum_{j \mid i \in \mathcal{U}_{j}} f_{t i j} & i \in \mathcal{I}, t \in \mathcal{T}, s \in \mathcal{S}
\end{array}
$$

where: 
(51) and (6) ensure respectively that the matched energy $p_{t i}^{s}$ will be greater than the instrumental price bid $q_{t i}$ and less than the total available energy not allocated to BC.

(17) and (8) guarantee respectively that the instrumental price bid will be greater than the minimum generation output of the unit and greater than the contribution of the unit to the FC coverage.

Please note that (21) together with (8) assures that $q_{t i}$ will be always nonnegative. The total generation level of a given unit $i, g_{t i}^{s}$, is defined as the addition of the allocated energy to the BC plus the matched energy of the DAM:

$$
g_{t i}^{s}=b_{t i}+p_{t i}^{s} i \in \mathcal{I}, t \in \mathcal{T}, s \in \mathcal{S}
$$

Contraints (11)-(9) assure that $g_{t i}^{s}$ will be always either zero or $g_{t i}^{s} \in\left[\underline{P}_{i}, \bar{P}_{i}\right]$, that is:

$$
\underline{P}_{i} u_{t i} \leq g_{t i}^{s} \leq \bar{P}_{i} u_{t i}, i \in \mathcal{I}, t \in \mathcal{T}, s \in \mathcal{S}
$$

Unit Commitment Constraints. This section includes the formulation for the unit commitment of the thermal units [2. The first two sets of constraints model the start-up and shut-down costs and the next ones control minimum operation and idle time for each unit. First, the start-up and shut-down costs are modeled:

$$
\begin{array}{ll}
c_{t i}^{u} \geq c_{i}^{o n}\left[u_{t i}-u_{(t-1) i}\right] & i \in \mathcal{I}, t \in \mathcal{T} \backslash\{1\} \\
c_{t i}^{d} \geq c_{i}^{o f f}\left[u_{(t-1) i}-u_{t i}\right] & i \in \mathcal{I}, t \in \mathcal{T} \backslash\{1\} \\
c_{t i}^{u}, c_{t i}^{d} \geq 0 & i \in \mathcal{I}, t \in \mathcal{T} \\
u_{t i} \in\{0,1\} & i \in \mathcal{I}, t \in \mathcal{T}
\end{array}
$$

The initial state of each thermal unit $i$ can be taken into account through the parameters $G_{i}$ and $H_{i}$ that represent, respectively, the number of the initial time periods along which the thermal unit must remain on $\left(G_{i}\right)$ or off $\left(H_{i}\right)$. These conditions are imposed by the following set of constraints:

$$
\sum_{j=1}^{G_{i}}\left(1-u_{j i}\right)=0 \quad \text { and } \quad \sum_{j=1}^{H_{i}} u_{j i}=0, \quad i \in \mathcal{I}
$$

Finally, the minimum up and down time, $t_{i}^{\text {on }}$ and $t_{i}^{\text {off }}$ are imposed, up to the periods $|\mathcal{T}|-\left(t_{i}^{o n}-1\right)$ and $|\mathcal{T}|-\left(t_{i}^{o f f}-1\right)$, through the following set of constraints:

$$
\begin{array}{ll}
\sum_{n=t}^{t+t_{i}^{o n}-1} u_{i n} \geq t_{i}^{o n}\left[u_{t i}-u_{(t-1) i}\right] & t=G_{i}+1, \ldots,|\mathcal{T}|-t_{i}^{o n}+1, i \in \mathcal{I} \\
\sum_{n=t}^{t+t_{i}^{\text {off }}-1}\left(1-u_{n i}\right) \geq t_{i}^{\text {off }}\left[u_{(t-1) i}-u_{t i}\right] & t=H_{i}+1, \ldots,|\mathcal{T}|-t_{i}^{\text {off }}+1 i \in \mathcal{I}
\end{array}
$$


and for the last $t_{i}^{o n}-1$ and $t_{i}^{o f f}-1$ time periods:

$$
\begin{array}{ll}
\sum_{n=t}^{|\mathcal{T}|}\left(u_{n i}-\left[u_{t i}-u_{(t-1) i}\right]\right) \geq 0 & t=|\mathcal{T}|-t_{i}^{\text {on }}+2, \ldots,|\mathcal{T}|, i \in \mathcal{I} \\
\sum_{n=t}^{|\mathcal{T}|}\left(1-u_{n i}-\left[u_{(t-1) i}-u_{t i}\right]\right) \geq 0 & t=|\mathcal{T}|-t_{i}^{\text {off }}+2, \ldots,|\mathcal{T}|, \quad i \in \mathcal{I}
\end{array}
$$

\subsection{Objective Function}

The quadratic function that gives the long-run expected profits of the GenCo after the participation in the DAM is:

$$
\begin{aligned}
\min \mathrm{E}_{\lambda^{D}}\left[C\left(u, c^{u}, c^{d}, g, p ; \lambda^{D}\right)\right]= & \sum_{t \in \mathcal{T}} \sum_{i \in \mathcal{I}}\left(c_{t i}^{u}+c_{t i}^{d}+c_{i}^{b} u_{t i}+\right. \\
& \left.+\sum_{s \in \mathcal{S}} P^{s}\left[\left(c_{i}^{l} g_{t i}^{s}+c_{i}^{q}\left(g_{t i}^{s}\right)^{2}\right)-\lambda_{t}^{D, s} p_{t i}^{s}\right]\right),
\end{aligned}
$$

where the right hand side of (20) is the on/off fixed cost of the unit commitment of the thermal units, deterministic and independent of the realization of the random variable $\lambda_{t}^{D, s}$ and (21) represents the expected value of the benefits from the DAM. The term between parenthesis corresponds to the expression of the quadratic generation costs associated to the total generation of the unit $g_{t i}^{s}$ while the last term, $\lambda_{t}^{D, s} p_{t i}^{s}$ computes the incomes from the DAM due to a value $p_{t i}^{s}$ of the matched energy.

Please note that the constant incomes from the BC and FC, i.e. $\sum_{k \in \mathcal{B}} \lambda_{k}^{B C} L_{k}^{B C}$ and $\sum_{t \in \mathcal{T}, j \in \mathcal{J}}\left(\lambda_{j}^{F C}-\bar{\lambda}_{t}^{D}\right) L_{j}^{F C}$, have been dropped from the objective function.

\subsection{Model (DAMB-FBC)}

The model defined so far can be represented as:

$$
(\mathrm{DAMB}-\mathrm{FBC}) \begin{cases}\min \mathrm{E}_{\lambda^{D}}\left[C\left(u, c^{u}, c^{d}, g, p ; \lambda^{D}\right)\right] \\ \text { s.t. } & \\ \text { Eq. (11) }-(41) & \text { BC and FC constraints } \\ \text { Eq. (15) }-(19) & \text { DAM and total gen. constraints } \\ \text { Eq. (11) }-(19) & \text { Unit commitment constraints }\end{cases}
$$

Model (DAMB-FBC) is the optimization problem associated with the two-stage stochastic programming problem with a set $\mathcal{S}$ of scenarios for the spot price $\lambda_{t}^{D}$ where $t \in \mathcal{T}$. This optimization problem is a convex MIQP with a well defined global optimal solution. 


\section{QBFC Method}

Model (DAMB-FBC) can be rewritten as the so-called Deterministic Equivalent Model (DEM)

$$
\begin{aligned}
\text { minimize } & c^{t} \delta+\sum_{s \in \mathcal{S}} P^{s} q^{s}\left(x, y^{s}\right) \\
\text { subject to }: & l_{a} \leq A\left[\begin{array}{l}
\delta \\
x
\end{array}\right] \leq u_{a}, \\
& l_{t}^{s} \leq T^{s}\left[\begin{array}{c}
\delta \\
x \\
y^{s}
\end{array}\right] \leq u_{t}^{s}, s \in \mathcal{S}, \\
& x \geq 0, \underline{y} \leq y^{s} \leq \bar{y}, s \in \mathcal{S}, \\
& \delta \in\{0,1\}^{n_{\delta}},
\end{aligned}
$$

where $\delta=u, \quad x=\left(c^{u}, c^{d}\right), \quad y=(g, p), \quad q(x, y)=b_{x}^{t} x+b_{y}^{t} y++y^{t} Q_{y y} y$, and $Q_{y y}$ being a diagonal matrix.

As is showed by 3 the compact representation (DEM) can be written as a splitting variable representation; i.e., $\delta$ and $x$ are respectively replaced by $\delta^{s}$ and $x^{s}$, for $s \in \mathcal{S}$. So, we have

$$
\begin{aligned}
(\mathrm{MIQP}) \text { minimize } & \sum_{s \in \mathcal{S}} P^{s}\left(c^{t} \delta^{s}+q^{s}\left(x^{s}, y^{s}\right)\right) \\
\text { subject to }: & l_{a} \leq A\left[\begin{array}{l}
\delta^{s} \\
x^{s}
\end{array}\right] \leq u_{a}, s \in \mathcal{S}, \\
& l_{t}^{s} \leq T^{s}\left[\begin{array}{l}
\delta^{s} \\
x^{s} \\
y^{s}
\end{array}\right] \leq u_{t}^{s}, s \in \mathcal{S}, \\
& x^{s} \geq 0, \underline{y} \leq y^{s} \leq \bar{y}, \quad \delta^{s} \in\{0,1\}^{n_{\delta}}, s \in \mathcal{S}, \\
\left(\mathrm{NAC}_{\delta}\right) & \delta^{s}-\delta^{s^{\prime}}=0, \forall s, s^{\prime} \in \mathcal{S}: s \neq s^{\prime}, \\
\left(\mathrm{NAC}_{x}\right) & x^{s}-x^{s^{\prime}}=0, \forall s, s^{\prime} \in \mathcal{S}: s \neq s^{\prime},
\end{aligned}
$$

where $\mathrm{NAC}_{\delta}$ and $\mathrm{NAC}_{x}$ are the nonanticipativity constraints.

In this method (DEM) is solved by using a Branch-and-Fix-Coordination scheme (BFC) for each scenario $s \in \mathcal{S}$ to fulfill the integrality condition (IC) on the variables $\delta$, so that the $\mathrm{NAC}_{\delta}$ are also satisfied when selecting branching nodes and branching variables by the Twin-Node-Families concept (TNF), which was introduced by [1].

A similar approach to that suggested in [3] is used in this work to coordinate the selection of the branching node and branching variable for each scenariorelated $\mathrm{BF}$ tree, such that the $\mathrm{NAC}_{\delta}$ are satisfied when fixing $\delta^{s}$, for all $s \in \mathcal{S}$, either to 1 or to 0 . A TNF integer set is a set of integer BF nodes (i.e. they verify $\mathrm{IC}$ ), one per $\mathrm{BF}$ tree, in which the $\mathrm{NAC}_{\delta}$ are verified. More details about this metodology can be found in 8 . 
When the number of scenarios is very hight, in order to gain computational efficiency we can take scenario clusters; i.e., instead a submodel for each scenario $s \in \mathcal{S}$ we can use a submodel $\left(\mathrm{MIQP}^{p}\right)$ for each scenario cluster $\mathcal{S}^{p} \subset \mathcal{S}$ with $p=1, \ldots, \widehat{p}$, where $\mathcal{S}^{p} \cap \mathcal{S}^{p^{\prime}}=\emptyset$, for all $p \neq p^{\prime}$, and $\cup_{p=1}^{\widehat{p}} \mathcal{S}^{p}=\mathcal{S}$,

$$
\begin{aligned}
&\left(\mathrm{MIQP}^{p}\right) \quad \text { minimize } \sum_{s \in \mathcal{S}^{p}} P^{s}\left(c^{t} \delta^{p}+q^{s}\left(x^{p}, y^{s}\right)\right), \\
& \text { subject to : } l_{a} \leq A\left[\begin{array}{l}
\delta^{p} \\
x^{p}
\end{array}\right] \leq u_{a}, \\
& \\
& l_{t}^{s} \leq T^{s}\left[\begin{array}{l}
\delta^{p} \\
x^{p} \\
y^{s}
\end{array}\right] \leq u_{t}^{s}, s \in \mathcal{S}^{p}, \\
& x^{p} \geq 0, \underline{y} \leq y^{s} \leq \bar{y}, s \in \mathcal{S}^{p}, \quad \delta^{p} \in\{0,1\}^{n_{\delta}},
\end{aligned}
$$

These submodels are linked by the NACs $\delta^{p}-\delta^{p^{\prime}}=0$ and $x^{p}-x^{p^{\prime}}=0$, for all $p, p^{\prime} \in\{1, \ldots, \widehat{p}\}$ such that $p \neq p^{\prime}$.

In order to gain computational efficiency we propose to use perspective cuts (PC) 52 to solve the quadratic subproblems in each node of the TNF. Then $\mathrm{MIQP}^{p}$ becomes:

$$
\begin{array}{ll}
\min & \sum_{s \in \mathcal{S}^{p}} P^{s}\left\{\left(b_{x}^{t} x+x^{t} Q_{x x} x\right)+\left(\sum_{i=1}^{n} v_{i}^{s}\right)\right\} \\
\text { s.t.: } & v_{i}^{s} \geq\left(2 q_{i i}^{s} \underline{y}_{i}+b_{i}^{s}\right) y_{i}^{s}+\left(c_{i}-q_{i i}^{s} \underline{y}_{i}^{2}\right) \delta_{i}^{s}, i \in\{1, \ldots, n\}, s \in \mathcal{S}^{p}, \\
& v_{i}^{s} \geq\left(2 q_{i i}^{s} \bar{y}_{i}+b_{i}^{s}\right) y_{i}^{s}+\left(c_{i}-q_{i i}^{s} \bar{y}_{i}^{2}\right) \delta_{i}^{s}, i \in\{1, \ldots, n\}, s \in \mathcal{S}^{p}, \\
& \text { Eq. (23) - (25). }
\end{array}
$$

These methods have been implemented in $\mathrm{C}++$ with the help of Cplex 12.1 to solve only the quadratic subproblems. In this work two algorithmic alternatives have been considered:

$\triangleright$ QBFC: coordination of $\delta$ in the TNF of the BF trees for clusters $p \in$ $\{1, \ldots, \widehat{p}\}$ without using PCs.

$\triangleright$ QBFC-PC: coordination of $\delta$ in the TNF of the BF trees for clusters $p \in$ $\{1, \ldots, \widehat{p}\}$ using PCs.

For our instances the number of scenarios in each cluster is the same, $\left|\mathcal{S}^{p}\right|=$ $|\mathcal{S}| / \widehat{p}$. Each cluster contains $\left|\mathcal{S}^{p}\right|$ consecutive scenarios, starting from the first one and following in natural order.

\section{Numerical Tests}

These instances are based on the liberalized electricity market model suggested in [2. In these problems $Q_{x x}$ is the zero matrix, as a result, when we use perspective cuts the subproblem to solve in each node is linear. The tests have been 
performed on HP with Intel(R) Core(TM)2 Quad CPU Q8300 2.50GHz 4 CPU under SUSE Linux Enterprise Desktop 11 (x86_64).

In Table $1|\mathcal{S}|$ means the number of scenarios, $|\mathcal{T}|$ the number of periods, "\# var" the number of continuous variables, "\# $\operatorname{var}_{P C F}$ " the number of continuous variables for the PC formulation, "\# bin" the number of binary variables, and "\# constr" the number of constraints for (DEM).

Table 1. Test problems

\begin{tabular}{lrllrrr}
\hline Prob. & $|\mathcal{S}|$ & $|\mathcal{T}|$ & $\#$ var \# & var \\
$P C F$ & $\#$ & bin \# constr \\
\hline P01 & 10 & 12 & 1296 & 1776 & 48 & 1788 \\
P02 & 20 & 12 & 2256 & 3216 & 48 & 3228 \\
P03 & 30 & 12 & 3216 & 4656 & 48 & 4668 \\
P04 & 40 & 12 & 4176 & 6096 & 48 & 6108 \\
P05 & 50 & 12 & 5136 & 7536 & 48 & 7548 \\
P11 & 10 & 24 & 2592 & 3552 & 96 & 3600 \\
P12 & 20 & 24 & 4512 & 6432 & 96 & 6480 \\
P13 & 30 & 24 & 6432 & 9312 & 96 & 9360 \\
P14 & 40 & 24 & 8352 & 12192 & 96 & 12240 \\
P15 & 50 & 24 & 10272 & 15072 & 96 & 15120 \\
\hline
\end{tabular}

For every problem $|\mathcal{F}|=|\mathcal{B}|=2$ and $|\mathcal{I}|=4$. If we use the PC formulation, the problem increases the number of variables in $m=|\mathcal{T}| \cdot|\mathcal{I}| \cdot|\mathcal{S}|$ and the number of constraints in $2 \cdot m$.

Table 2. Computational results: CPU-times

\begin{tabular}{llrrrr}
\hline Prob. $\widehat{p}$ & QBFC & QBFC-PC ratio \# PC \\
\hline P01 & 2 & 10.1 & 3.4 & 0.34 & 280 \\
P02 & 4 & 18.7 & 8.7 & 0.47 & 825 \\
P03 & 5 & 2153.0 & 39.8 & 0.02 & 1685 \\
P04 & 5 & 50.0 & 45.1 & 0.90 & 1491 \\
$\mathrm{P} 05$ & 5 & 113.7 & 19.5 & 0.17 & 1276 \\
$\mathrm{P} 11$ & 2 & 86.8 & 27.4 & 0.32 & 513 \\
$\mathrm{P} 12$ & 4 & 469.7 & 50.3 & 0.11 & 1821 \\
$\mathrm{P} 13$ & 5 & 687.3 & 176.6 & 0.26 & 3454 \\
$\mathrm{P} 14$ & 5 & 1198.0 & 276.7 & 0.23 & 4239 \\
$\mathrm{P} 15$ & 5 & 1190.9 & 246.3 & 0.21 & 2592 \\
\hline
\end{tabular}

In Table 2 below the headings QBFC are the times in CPU-seconds used for solving problems with the number of scenario cluster given below the heading $\widehat{p}$ and by solving the quadratic subproblem $\mathrm{QP}^{p}$ for each node using Cplex. Column QBFC-PC gives us the CPU-seconds and indicates that the quadratic subproblems $\mathrm{QP}^{p}$ have been solved by using perspective cuts, which means that instead of solving a quadratic problem $\mathrm{QP}^{p}$ in each node of a TNF for $p \in$ 
$\{1,2, \ldots, \widehat{p}\}$, a linear problem is solved. Also, "ratio" $=\frac{\mathrm{QBFC}-\mathrm{PC}}{\mathrm{QBFC}}$ gives us the ratio of CPU-times. Note that the running time with $\mathrm{PC}$ is a $30 \%$ of the running time without PC (average). The last column, "\# PC", means the number of perspective cuts generated in each test.

\section{Conclusions}

We have presented an Optimal Bidding Model for a price-taker generation company operating both in the MIBEL Derivatives and Day-Ahead Electricity Market (DAMB-FBC). The model developed finds the optimal bid for the spot market, the optimal allocation of the physical futures and bilateral contracts among the thermal units and the unit commitment following in detail the MIBEL rules. The (DAMB-FBC) has been solved both with the standard BFC method and with a $\mathrm{PC}$ variation which reduces the running time to a $30 \%$ on the average.

\section{References}

1. Alonso-Ayuso, A., Escudero, L.F., Ortuño, M.T.: BFC, a branch-and-fix coordination algorithm framework for solving some types of stochastic pure and mixed 0-1 programs. European Journal of Operational Research 151, 503-519 (2003)

2. Corchero, C., Mijangos, E., Heredia, F.J.: A new optimal electricity market bid model solved through perspective cuts. TOP (2011) (published online 2011), doi:10.1007/s11750-011-0240-6

3. Escudero, L.F., Garín, M., Merino, M., Pérez, G.: A general algorithm for solving two-stage stochastic mixed 0-1 first-stage problems. Computers \& Operations Research 36, 2590-2600 (2009)

4. Escudero, L.F., Garín, M., Merino, M., Pérez, G.: An algorithmic framework for solving large-scale multistage stochastic mixed 0-1 problems with nonsymmetric scenario trees. Computers \& Operations Research 39(5), 1133-1144 (2012)

5. Frangioni, A., Gentile, C.: Perspective cuts for a class of convex 0-1 mixed integer programs. Mathematical Programming 106, 225-236 (2006)

6. Frangioni, A., Gentile, C.: A computational comparison of reformulations of the perspective relaxation: SOCP vs. cutting planes. Operations Research Letters 37, 206-210 (2009)

7. Hull, J.C.: Options, futures and other derivatives, 5th edn. Prentice-Hall International, Englewood Cliffs (2002)

8. Mijangos, E.: An Algorithm for Two-Stage Stochastic Quadratic Problems. In: Hömberg, D., Tröltzsch, F. (eds.) CSMO 2011. IFIP AICT, vol. 391, pp. 181-191. Springer, Heidelberg (2013)

9. Tawarmalani, M., Sahinidis, N.: Semidefinite relaxations of fractional programs via novel convexification techniques. Journal of Global Optimization 20, 137-158 (2001) 